\title{
An Entropy based Model for Examination of Social Media Data Mining for Marketing Intelligence
}

\author{
N. Naveen, S. Suresh, P. Karunakar Reddy
}

\begin{abstract}
Social Websites Provides Platform for to Deliver Customer Opinion in the form of comments. It is also Highly Impossible to get the huge comments throughout the World; in this Paper we proposed the Concept of Customer Review and Ranking of the Product in the Marketing for this us used an Algorithm of re-ranking technique. Entropy Based Model supports for review and also used for reviews along with ranking Our algorithm Gives Better results comparing with the Other Existed Algorithms.The Output results gives the Better Marketing Intelligence Strategy that will supports customer to select good in the Marketing. And also supports for Blocking Website in the Marketing Who are not providing customer not satisfied Product in the Market.
\end{abstract}

Key-Words:-Social Media, Data Mining, Ranking, Search strategy.

\section{I.INTRODUCTION}

Internet based life are progressively being utilized in mainstream researchers as a key wellspring of information to help comprehend differing regular and social wonders, and this has incited the advancement of a wide scope of computational data mining instruments that can separate learning from web based life for both post-hoc and constant investigation.

Due to that accessibility of an open API that empowers those sans cosset gathering of a quite a few information, twitter need transformed under a primary data hotspot for such examinations [3]. Hosting twitter Concerning illustration in turn sort data source, masters bring investigated the change from claiming instruments to consistent design examination [2], [6] alternately early ID number for newsworthy events [1], in the same way that under deliberate methodologies for seeing the incline communicated Toward customers towards a destination [4], [6], [2], alternately all Decision on a specific purpose [5].

Be that as it may, twitter majority of the data necessities trustworthy fact subtleties that might enable a representative test about customers on make assembled and

Revised Manuscript Received on September 22, 2019.

Dr.N.Naveen, Assistant Professor, Dept. of CSE

Dr. S.Suresh, Associate Professor, Dept. of CSE, Pragati engineering college

Dr. P.Karunakar Reddy, Assistant Professor, Dept. of CSE, Ashoka institute of engineering and technology also a consideration ahead a specific customer subgroup [3], or other unequivocal applications, to example, setting up those dependability of information presented [4]. Electronic finding of web based term socioeconomics might make helpful, "around others, to extend demographically careful web based an aggregation investigations that would guided through investigations [6]. A standout amongst the missing fact subtleties is a client's country about source, which we consider here. Those principle decision afterward to the master is on endeavor should accumulate such detail qualities When endeavoring those wanted examination.

This has inspired a developing assortment of research as of late taking a gander at various methods for deciding consequently the client's nation of birthplace or potentially - as an intermediary for the previous - the area from which tweets have been posted [1]. The vast majority of the past research in deducing tweet Geo area has grouped tweets by area inside a restricted land territory or nation; these can't make connected clearly will an unfiltered stream the place tweets from whatever territory or country will be viewed. The couple instances that need figured out how An overall aggregation for tweets need used an expansive course of action from claiming highlights that can't practically be divided Previously, An continuous, spilling setting (e. G., customer tweeting history alternately casual organizations) [11], What's more need been compelled on a decided situated of around the world urban groups in the same way that should English tweets. This infers they utilization ground To names to pre-channel tweets starting from separate locale alternately conceivably composed clinched alongside dialects other than English. Those classifier In view of this pre-separated dataset might not a chance to be appropriate will An twitter stream the place every tweet ought further bolstering a chance to be geo discovered.

A capacity should request tweets Toward territory progressively is Dire to provisions abusing web based an aggregation refreshes as social sensors that enable accompanying subjects Furthermore discovering crazy regarding region unequivocal inclining themes, climbing events Furthermore softening news. Express utilizations about an ongoing, nation level

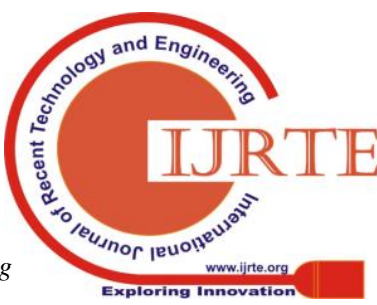


tweet geolocation framework incorporate nation explicit slanting subject identification or following slant towards a point separated by nation. To the best of our insight, our work is the first to manage global tweets in any language, utilizing just those highlights present within the substance of a tweet and its related metadata.

We additionally supplement past work by researching the extent to which a classifier prepared on chronicled tweets can be utilized adequately on recently gathered tweets. Persuaded by the need to build up an application to distinguish the drifting subjects inside a particular country1, here we file the headway of a classifier that might geo Figure tweets Toward country about origin progressively. Provided for that inside this circumstance it isn't useful will assemble additional data to that immediately receptive from those twitter stream [11], we examine the support about eight tweet-intrinsic highlights, which need aid constantly on immediately approachable starting with An tweet item Likewise recouped starting with those twitter API, for choosing its geolocation.

perform arrangement utilizing every one of the highlights alone, yet in addition in highlight mixes. We investigate the capacity to play out the characterization on upwards of 217 nations, or in a diminished subset of the main 25 nations, as made a decision by tweet volume. The utilization of two datasets, gathered in October 2014 and October 2015, gives extra understanding into whether verifiable Twitter information can be utilized to characterize new instances of tweets. These two datasets with more than 5 million nation coded tweets are openly accessible. Our philosophy empowers us to play out an exhaustive investigation of tweet geo area, uncovering experiences into the best approaches for an exact nation level area classifier for tweets. We find that the utilization of a solitary element like substance, which is the most generally utilized component in past work, doesn't do the trick for a precise characterization of clients by nation and that the mix of various highlights prompts significant improvement, beating the best in class continuous tweet geo area classifier; this improvement is especially show when utilizing metadata like the client's self-revealed area just as the client's genuine for the main 25 nations as far as tweet volume, investigating how various highlights lead to ideal order for various nations, just as examining restrictions when dealing with probably the most testing nations. We show that country-level order of an unfiltered Twitter stream is testing.

It requires cautious plan of a classifier that uses a are promising enough on account of various nations, empowering further investigation into better grained geo area of worldwide tweets. Situations where nation level geo area is all the more testing incorporate English and Spanish name. We likewise play out a for each nation investigation proper blend of highlights. Our outcomes at the nation level

talking nations, which are more diligently to distinguish due to their various shared characteristics. In any case, our experiments show that we can accomplish F1 scores above $80 \%$ in numerous of these cases given the decision of a suitable combination of highlights, just as a general presentation above $80 \%$ in terms of both small scale exactness and full scale precision for the top 25 nations

\section{RELATED WORK}

A few analysts have been chipping away at conclusion grouping, otherwise called extremity characterization for online item surveys, so as to anticipate whether buyers like an item or not, founded on the audits. Hatzivassiloglou et al. proposed a strategy to anticipate the semantic direction of descriptive words by an administered learning calculation [1]. Competition exhibited an unaided learning calculation to characterize surveys as prescribed or not suggested by an-investigating the semantic direction dependent on shared data [7]. In [11] and [4], the creators proposed characterization ways to deal with group surveys as positive or negative.The impact of online item audits on the item deals is additionally a fascinating examination territory. In [5], the creators found that the nature of surveys has constructive outcome on productsales, and shoppers buy aims increments with thequantity of item audits. Hu et al. seen that buyers considered the audit's evaluations, yet in addition thecontextual data, for example, commentators' notoriety [3].They likewise found that the effect of online surveys on deals decreases after some time. Assessing the quality and support of audits orposts on web gatherings is another examination space.

Over [2], Kim et al. Passed on a methodology with characteristically overview re-see convenience. They used SVM should get ready that framework and discovered that that period of the survey, the unigrams and the result rating is those practically important highlights. Weimer et al. Recommended An programmed figuring to review those personal satisfaction from can presents clinched alongside web dialogs using highlights, for example, surface, lexical, syntactic, gathering explicit and comparative highlights [9]. In [8], Weimer and Iryna broadened the technique into three datasets and saw that the SVM grouping performs well. In this paper, another entropy-based methodology by scoring the support of online item audits is investigated. With this proposed approach, online item surveys could be evaluated and positioned.

\section{III.PROPOSED APPROACH}

Our work centers around examining item audits in order to discover high caliber and supportive surveys. In this area, we

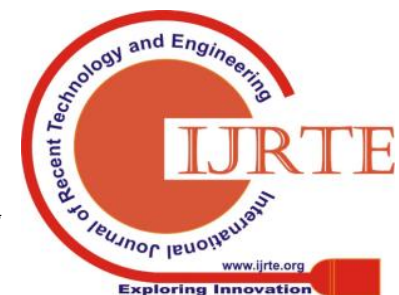


discuss how to evaluate the audits' accommodation and build up the supportiveness work.

\subsection{ENTROPY AND INFORMATION GAIN}

In the work by Pang et al. [4], the creators detailed that the best outcome was acquired by utilizing Boolean estimations of unigram features. Propelled by this methodology, we use bag of words model to speak to content and fabricate our language model. Each element is a constant stemmed word and the value of this element is a Boolean estimation of the event of the word on the review. We present the Shannon's data entropy idea [6] to gauge the measure of data in surveys. In the area of order, the entropy can be stretched out as pursues: Let $\mathrm{S}=\{\mathrm{s} 1, \mathrm{~s} 2, \ldots \ldots \mathrm{sq}\}$ be the arrangement of classifications in the review space. The normal data expected to classify a audit is:

$$
H(S)=\sum_{i=1}^{m} P_{r}\left(s_{i}\right) \log P_{r}\left(s_{i}\right)
$$

The average amount of information contributed by a term 1 in

$$
\text { the class Si will be: } I I(S \mid t)--\sum_{i=1}^{m} P_{r}\left(s_{i} \mid t\right) \log P_{r}\left(s_{i} \mid t\right)
$$

Data increase is gotten from entropy and can be comprehended as the normal entropy decrease by knowing the presence of a term $\mathrm{t}$.

$$
\mathrm{G}(\mathrm{t})=\mathrm{H}(\mathrm{s}) \mathrm{H}(\mathrm{s} \backslash \mathrm{t}) \quad 4
$$

It is frequently utilized as a term's integrity basis in the field of AI [10] and regularly utilized as a component choice strategy in content grouping. $\operatorname{In}[10]$, the data increase of term L was expanded and characterized as pursues

$$
G(t)=-\sum_{i=1}^{q} P_{r}\left(s_{i}\right) \log P_{r}\left(s_{i}\right)+P_{r}(t) \sum_{i=1}^{q} P_{r}\left(s_{i} \mid t\right) \log P_{r}\left(s_{i} \mid t\right)
$$

\section{IV.LITERATURE SURVEY}

"TVDc: Managing Security in the Trusted Virtual Datacenter", S. Berger et al., Virtualization innovation is ending up progressively basic in datacenters, since it takes into consideration collocation of various remaining burdens, comprising of working frameworks, middleware and applications, in various virtual machines (VMs) on shared physical equipment stages. In any case, when combined with the simplicity of VM relocation, this pattern expands the potential surface for security assaults. Further, the improved administration of VMs, including creation, cloning and movement, makes it basic to screen and ensure the honesty of programming segments running inside VMs.

This paper introduces the IBM Trusted Virtual Datacenter (TVDc) innovation created to address the requirement for solid disengagement and honesty ensures, hence altogether upgrading security and frameworks the board abilities, in virtualized conditions. It connotes the primary exertion to join confided in processing advancements legitimately into virtualization and frameworks the board programming. We present and talk about different parts that establish TVDc: the Trusted Platform Module (TPM), the virtual TPM, the IBM hypervisor security engineering (sHype) and the related frameworks the executives programming.

\section{A Framework for Building Privacy-Conscious} Composite Web Services, W. Xu, V.N. Venkatakrishnan, R. Sekar, and I.V. Ramakrishnan,

The quick development of web applications has incited expanding enthusiasm for the territory of composite web benefits that include a few specialist organizations. The potential for such composite web administrations can be acknowledged just if buyer protection concerns are acceptably tended to. In this paper, we propose a system that tends to purchaser protection worries with regards to exceptionally adjustable composite web administrations. Our methodology includes administration makers trading their terms-of-utilization with customers as "models". Our structure gives computerized systems to checking these models at the purchaser site for consistence of customer protection strategies. In case of a strategy infringement, our system underpins programmed age of "commitments" that the shopper creates for the composite administration. These commitments are consequently implemented through a powerful program investigation approach on the web administration sythesis code. We represent our methodology with the usage of two model administrations.

\section{Towards Standardized Web Services Privacy Technologies, P.C.K. Hung, E. Ferrari, and B. Carminati}

A Web administration is characterized as a self-governing unit of use rationale that gives either some business usefulness or data to different applications through an Internet association. Web administrations depend on a lot of XML principles, for example, widespread depiction, disclosure and reconciliation (UDDI), Web administrations portrayal language (WSDL), and basic article get to convention (SOAP). As of late there are expanding requests and dialogs about Web administrations security innovations in the business and research network. When all is said in done, protection arrangements depict an association's information rehearses what data they gather from people (e.g., shoppers) and what (e.g., purposes) they do with it. To empower security assurance for Web administration shoppers over different areas and administrations, the World Wide Web Consortium (W3C) distributed an archive called "Web administrations engineering (WSA) necessities" that characterizes 
some particular protection prerequisites for Web benefits as a future research subject. As of now, there is still no institutionalized Web administrations protection innovation. This paper quickly reviews the exploration issues of Web administrations security advancements.

\section{Managing and Securing Web Services with VPNs, L.}

\section{Alchaal, V. Roca, and M. Habert}

Web Services comprise a lot of advances that many accept will change the Web correspondence scene inside the following couple of years. They offer institutionalized and simple correspondences for circulated frameworks over the Internet. Anyway their dynamic and circulated nature requires a well-overseen framework, and pending security issues counteract their far reaching selection. In the interim there is a major anger toward the utilization of Virtual Private Networks (VPNs) to verify interchanges in a financially savvy condition like the Internet. In this paper we disclose how to consolidate these two advances in another ground-breaking half breed model that: (1) empowers a simple administration of Web administrations, (2) gives Web administrations security on account of the utilization of dynamic and programmable VPNs, and (3) stays straightforward and completely coordinated

\section{Software Integrity Protection Using Timed Executable}

Agents, J. Garay and L. Huelsbergen

We present a product conspires for securing the trustworthiness of registering stages utilizing Timed Executable Agent Systems (TEAS). A believed challenger issues a confirmed test to a maybe degenerate responder. New is that the issued test is an executable program that can conceivably process any capacity on the responder. The responder must process not just the right worth inferred by the specialist, yet in addition must finish this calculation inside time limits recommended by the challenger. Programming based authentication plans have been proposed previously - new abilities acquainted in TEAS furnish implies with alleviate the current deficiencies of such proposed methods. TEAS are general and can be adapted to numerous applications for which framework trustworthiness is to be tried.

\section{V.CONCLUSION AND FEATURE WORK}

This paper proposes a way to deal with model Social Media Data Mining. By applying this model, online item audits can be ordered and positioned dependent on their score esteems. This will enable clients to complete their data search and basic leadership process all the more effectively. And also Easy to filter the good Product using Ranking .Surveys from other item classes and significantly bigger survey sets will be examined later on. Future research would likewise take different elements that may influence the nature of audits into thought (e.g., when the survey is distributed, how the purchasers have evaluated the item, and the quantity of highlights specified in the survey and so on.). Our model can likewise be acquainted with a web index to improve the precision of list items. We are gathering more information to look at these conceivable research bearings.

\section{REFERENCES}

1. V. Hatzivassiloglou and K. R. McKeown. Predicting the semantic orientation of adjectives. In Proceedings of the eighth conference on European chapter of the Association for Computational Linguistics, pages 174-181, Morristown, NJ, USA, 1997. Association for Computational Linguistics.

2. S.-M. Kim, P. Pantel, T. Chklovski, and M. Pennacchiotti. Automatically assessing review helpfulness. In Proceedings of the 2006 Conference on Empirical Methods in Natural Language Processing, pages 423-430, Sydney, Australia, July 2006. Association for Computational Linguistics.

3. L. L. Nan Hu and J. J. Zhang. Do online reviews affect product sales? the role of reviewer characteristics and temporal effects. Information Technology and Management, 2008. [4] B. Pang, L. Lee, and S. Vaithyanathan. Thumbs up?: sentiment classification using machine learning techniques. In EMNLP '02: Proceedings of the ACL-02 conference on Empirical methods in natural language processing, pages 79-86, Morristown, NJ, USA, 2002. Association for Computational Linguistics.

4. D.-H. Park, J. Lee, and I. Han. The effect of on-line consumer reviews on consumer purchasing intention: The moderating role of involvement. Int. J. Electron. Commerce, 11(4):125-148, 2007.[6] C. E. Shannon. A mathematical theory of communication. SIGMOBILE Mob. Comput. Commun. Rev., 5(1):3-55,2001.

5. P. Turney. Thumbs up or thumbs down? semantic orientation applied to unsupervised classification of reviews.

6. I. Weimer, Markus; Gurevych. Predicting the perceived quality of web forum posts. Proceedings of the Conference on Recent Advances in Natural Language Processing (RANLP), 2007.

7. M.Weimer, I. Gurevych, and M.M“uhlh“auser. Automatically assessing the post quality in online discussions on software. In Proceedings of the 45th Annual Meeting of the Association for Computational Linguistics Companion Volume Proceedings of the Demo and Poster Sessions, pages 125-128, Prague, Czech Republic, June 2007. Association for Computational Linguistics.

8. Y. Yang and J. O. Pedersen. A comparative study on feature selection in text categorization. In Proceedings of the Fourteenth International Conference on Machine Learning, pages 412-420, San Francisco, CA, USA, 1997. Morgan Kaufmann Publishers Inc.

9. H. Yu and V. Hatzivassiloglou. Towards answering opinion questions: separating facts from opinions and identifying the polarity of opinion sentences. In Proceedings of the 2003 conference on Empirical methods in natural language processing, pages 129-136, Morristown, NJ, USA, 2003. Associationfor Computational Linguistics. 